\title{
Brain-derived neurotrophic factor gene polymorphisms are associated with coronary artery disease-related depression and antidepressant response
}

\author{
YONG-QIANG LIU ${ }^{1}$, GUO-BAO SU ${ }^{1}$, CHANG-HONG DUAN ${ }^{1}$, JUN-HUA WANG ${ }^{1}$, \\ HAI-MEI LIU ${ }^{1}$, NAN FENG ${ }^{1}$, QING-XI WANG ${ }^{2}$, XU-EN LIU ${ }^{2}$ and JIE ZHANG ${ }^{1}$ \\ ${ }^{1}$ Department of Cardiothoracic Surgery, The First Affiliated Hospital of Xinxiang Medical University, Weihui, Henan 453100; \\ ${ }^{2}$ The Third Department of Psychiatry, Henan Mental Health Center, Xinxiang, Henan 453002, P.R. China
}

Received September 21, 2013; Accepted May 2, 2014

DOI: $10.3892 / \mathrm{mmr} .2014 .2638$

\begin{abstract}
Depression is a well-established risk factor for cardiac morbidity and mortality in patients with coronary artery disease (CAD). Previous studies have demonstrated that the level of brain-derived neurotrophic factor (BDNF) is decreased in depressed patients and this depletion may be reversed by antidepressants. Several recent studies have suggested that BDNF is involved in the pathogenesis of CAD. The aim of the present study was to investigate the possible association between seven single nucleotide polymorphisms (SNPs) of the BDNF gene (SNPs; rs16917204, rs6265, rs7103873, rs16917237, rs56164415, rs13306221 and rs10767664) and coronary artery disease-related depression (CAD-D). In the present study, 616 CAD patients without depression (CAD-nD) and 155 patients with CAD-D were recruited, and the response to an eight week sertraline antidepressant treatment regimen was also evaluated. The results demonstrated that a significant association existed between the SNP rs6265, located in exon 4 of the BDNF gene, and CAD-D $\left[\chi^{2}=9.634, \mathrm{P}=0.002\right.$, odds ratio $(\mathrm{OR})=1.486,95 \%$ confidence interval $(\mathrm{CI})=1.156-1.910]$. Another potential association was observed for rs13306221 $\left(\chi^{2}=5.194, \mathrm{P}=0.023, \mathrm{OR}=2.139\right.$, $95 \% \mathrm{CI}=1.096-4.175)$ in the promoter region of the BDNF gene. Strong linkage disequilibrium was observed in block 1
\end{abstract}

Correspondence to: Dr Jie Zhang, Department of Cardiothoracic Surgery, The First Affiliated Hospital Xinxiang Medical University, Jianshe Road 388, Weihui, Henan 453100, P.R. China

E-mail: liuyongqiangys@163.com

Abbreviations: BDNF, brain-derived neurotrophic factor; CAD, coronary artery disease; CAD-D, coronary artery disease-related depression; CAD-nD, CAD without depression; HAMD, Hamilton Depression Rating Scale; HWE, Hardy-Weinberg equilibrium; MD, major depression; SSRIs, selective serotonin reuptake inhibitors; SNPs, single nucleotide polymorphisms; UTR, untranslated region

Key words: brain-derived neurotrophic factor, coronary artery disease-related depression, polymorphism, sertraline (rs16917204, rs6265; D'>0.9). However, there was no evidence of a significant linkage disequilibrium between the seven SNPs in our sample population. Additionally, carriers of the A allele of rs6265 exhibited improved responses to the sertraline treatment $\left(\chi^{2}=8.942, \mathrm{P}=0.003, \mathrm{OR}=2.136,95 \% \mathrm{CI}=1.293-3.528\right)$. To the best of our knowledge, these results demonstrate, for the first time, the presence of a significant association between BDNF rs6265 and CAD-D, the identification of which may facilitate early diagnosis of CAD-D in the future.

\section{Introduction}

Coronary artery disease (CAD) is a leading cause of death in developed countries, with an annual mortality rate of $>7$ million (1). Up to $20 \%$ of patients with CAD meet the Diagnostic and Statistical Manual of Mental Disorders-IV criteria (DSM-IV) for major depression (MD), which is reported to be associated with adverse cardiac outcomes $(1,2)$.

Brain-derived neurotrophic factor (BDNF), a member of the neurotrophin family of growth factors, promotes survival, differentiation and maintenance of neurons in the nervous system (3). Previous studies have revealed the importance of BDNF in the development of the cardiac vasculature, including evidence that BDNF promotes angiogenesis (4), enhances vascular flow and regulates revascularization of ischemic tissue (5). Recently, clinical studies have demonstrated that BDNF is associated with coronary atherosclerosis and unstable angina, and experimental studies have revealed that BDNF may contribute to plaque instability in CAD, since it enhances the activity of local nicotinamide adenine dinucleotide (phosphate) oxidase and superoxide production (6). In addition to its association with CAD, BDNF is also reported to be associated with the pathogenesis of MD (7). For example, one study identified that in animal models of depression, the level of BDNF was decreased during hippocampal formation (8). Furthermore, the mRNA level of BDNF is lower in the hippocampus and anterior cingulate cortex in MD patients compared with healthy controls (9).

A number of single nucleotide polymorphisms (SNPs) have been identified in the BDNF gene, including rs13306221 in the promoter region (10), rs56164415 in intron 2 (11), rs6265 
in exon 4 (12) and rs16917204 in the 3'-untranslated region (UTR) (13). All of these SNPs are reported to be associated with the expression of the BDNF protein. A recent meta-analysis revealed that the A allele carriers had an increased risk for geriatric depression (14). The putative promoter region contains a SNP rs13306221 (712 bp upstream of the first exon) that is associated with substance dependence (10) and MD patients (15). The rs16917204 polymorphism in the 3'-UTR of BDNF has also been associated with Alzheimer's disease-related depression (16). Previously, a case-control study demonstrated evidence of an association between the rs6265 polymorphism and coronary artery disease-related depression (CAD-D) (17).

In view of the crucial role of the BDNF gene in CAD and MD, the aim of this study was to elucidate the potential association of seven BDNF polymorphisms with CAD-D and treatment response to selective serotonin reuptake inhibitors (SSRIs).

\section{Materials and methods}

Subjects. The subjects were consecutively recruited from The First Hospital Affiliated to Xinxiang Medical College between May 2006 and March 2013, including 616 CAD without depression (CAD-nD) patients (the mean $\pm \mathrm{SD}$ age, $61.6 \pm 5.6$ years). The patients with CAD-D were recruited as follows: First, the CAD patients in The First Hospital Affiliated to Xinxiang Medical College received the laboratory examinations including, coronary angiography, electrocardiogram (EKG), blood tests and/or stress tests. The interview and response was also performed on the patients. Coronary angiography was conducted in all patients. Inclusion criteria were as follows: (i) Patients had at least one diseased vessel $(\geq 50 \%$ stenosis) in the coronary angiograph; (ii) patients with chronic stable angina pectoris (SAP) had effort ischemic symptoms which lasted for at least three months and a positive stress test; (iii) patients with unstable angina pectoris (UAP) had chest discomfort or other ischemic symptoms at rest or in a progressive crescendo pattern without a rise in biomarkers of myocardial necrosis; (iv) patients presented with transient ST-T segment depression and T-wave inversion, with a typical elevation in biomarkers of myocardial necrosis; (v) patients with acute myocardial infarction (AMI) presented with ischemic symptoms and with ST-segment elevations persistent and ST-segment elevation on EKG. The majority of these patients would exhibit a typical elevation in the biomarkers of myocardial necrosis. Secondly, the MD of the CAD patients was diagnosed with structured clinical interviews, based on DSM-IV criteria for MD. Depressive symptoms were classified using the 24-item Hamilton Depression Rating Scale (HAMD). The patients who had a minimum score of 20 on the HAMD scale were included. Finally, 155 subjects with CAD-D (the mean \pm SD age, 62.2 \pm 6.8 years) met the DSM-IV criteria. The present study was performed according to the Guidelines of the Medical Ethical Committee of Xinxiang Medical College. Written informed consent was obtained from all individuals involved.

Treatment. The treatment response of patients was evaluated using HAMD. Participants who exhibited at least three
Table I. Demographic and clinical characteristics in CAD-nD patients $(n=616)$ and CAD-D patients $(n=155)$.

\begin{tabular}{lcc}
\hline Variable & CAD-nD & CAD-D \\
\hline Age (years) & $61.6 \pm 5.6$ & $62.2 \pm 6.8$ \\
Education (years) & $11.6 \pm 3.3$ & $11.8 \pm 3.2$ \\
Family history (yes) & $91(14.77)$ & $27(17.42)$ \\
LVEF (\%) & $59.7 \pm 10.4$ & $60.6 \pm 9.6$ \\
Ccr (ml/min) & $73.6 \pm 20.5$ & $75.6 \pm 18.3$ \\
hs-CRP (mg/dl) & $4.1 \pm 3.6$ & $4.4 \pm 3.5$ \\
TC (mmol/l) & $4.3 \pm 1.4$ & $4.5 \pm 1.1$ \\
TG (mmol/l) & $1.8 \pm 1.2$ & $1.7 \pm 1.0$ \\
HDL-C (mmol/l) & $1.3 \pm 0.5$ & $1.6 \pm 1.0$ \\
LDL-C (mmol/l) & $2.9 \pm 1.2$ & $2.5 \pm 1.1$ \\
With suicide attempt $^{\mathrm{a}}$ & $5.1 \pm 0.2$ & $8.3 \pm 0.2$ \\
HAMD $^{\mathrm{a}}$ & $14.5 \pm 6.1$ & $24.6 \pm 6.6$ \\
\hline
\end{tabular}

${ }^{\mathrm{a}} \mathrm{AD}-\mathrm{D}$ compared with $\mathrm{AD}-\mathrm{nD}$, differences reaching statistical significance are $\mathrm{P}<0.01$. CAD-nD, coronary artery disease without depression; CAD-D, coronary artery disease-related depression; LVEF, left ventricular ejection fraction; $C c r$, creatinine clearance rate; hs-CRP, high-sensitivity C-reactive protein; TC, total cholesterol; TG, triglyceride; HDL-C, high density lipoprotein-cholesterol; LDL-C, low density lipoprotein-cholesterol; HAMD, Hamilton Depression Rating Scale.

symptoms according to the DSM-IV criteria $(n=155)$ received antidepressant treatment with sertraline $(150 \mathrm{mg} /$ day for 8 weeks). Five patients did not complete the sertraline treatment. On the contrary, $616 \mathrm{CAD}-\mathrm{nD}$ patients received standard care but no antidepressants. Patient response was assessed at the end of the 8 week treatment. The patients with a HAMD score at $\leq 7$ or a $50 \%$ improvement were considered to be remittent.

Genotyping. The relative genomic locations of the BDNF gene with a genomic size of $67.2 \mathrm{~kb}$ and selected SNPs are illustrated in Fig. 1. Peripheral blood was drawn from a vein into a sterile tube containing ethylenediamine tetraacetic acid (EDTA). Plasma samples were stored at $-20^{\circ} \mathrm{C}$. Genomic DNA was extracted from the frozen peripheral blood samples using a QIAamp Blood Mini kit (Qiagen Inc., Valencia, CA, USA) according to the manufacturer's instructions. Genotyping was performed for all SNPs using the MassARRAY platform (Sequenom, San Diego, CA, USA). Primer extension and PCR were conducted according to the manufacturer's instructions, using iPLEX enzyme (Sequenom) and HotStarTaq DNA polymerase (Qiagen, Hilden, Germany). The resulting spectra were processed with the MassARRAY RT software (version 3.0.0.4) and genotype data was analyzed using the MassARRAY Typer software (version 3.4; Sequenom).

Statistical analysis. The data were analyzed using SPSS 16.0 (SPSS, Inc, Chicago, IL, USA). Hardy-Weinberg equilibrium (HWE) for the distributions of genotypes was estimated by chi-square $\left(\chi^{2}\right)$ tests. The potential association between CAD-D and each polymorphism was analyzed using Fisher's 


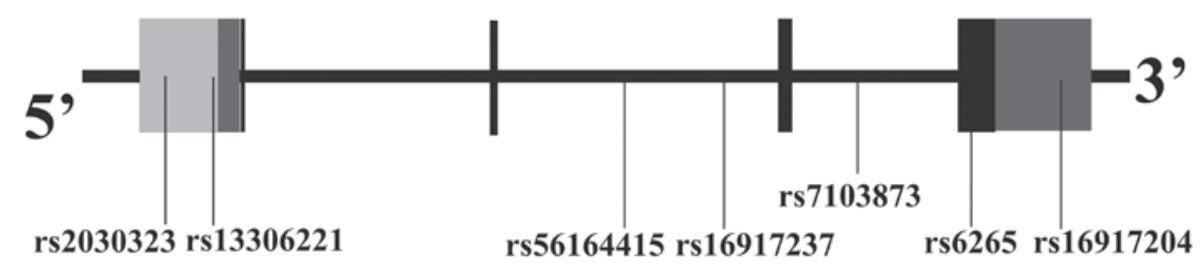

\begin{tabular}{ll|l} 
Promoter Exon Intron UTR & SNPs with MAF greater 5\%
\end{tabular}

Figure 1. Gene structure of human BDNF, illustrating the relative positions of the seven SNPs used in the present study. SNP, single nucleotide polymorphism; UTR, 3'-untranslated region; MAF, minor allele frequency.

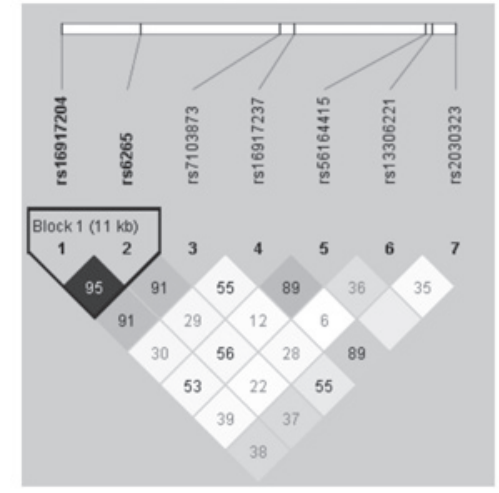

Controls ( $\left.\mathbf{D}^{\prime}\right)$

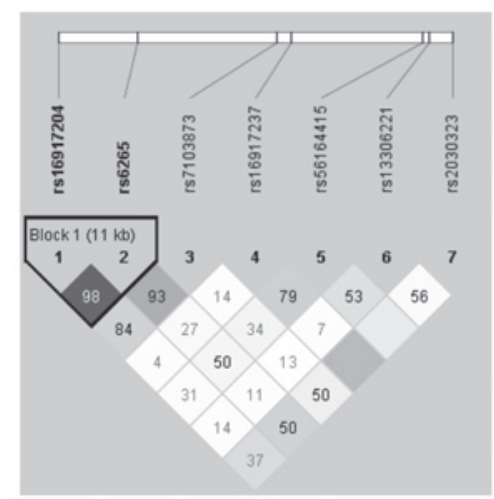

Cases ( D')

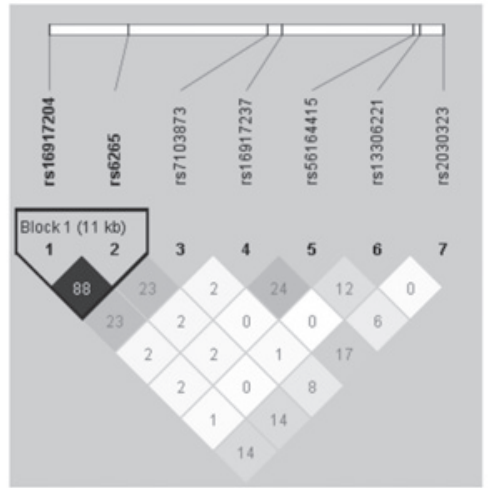

Controls $\left(\mathbf{r}^{2}\right)$

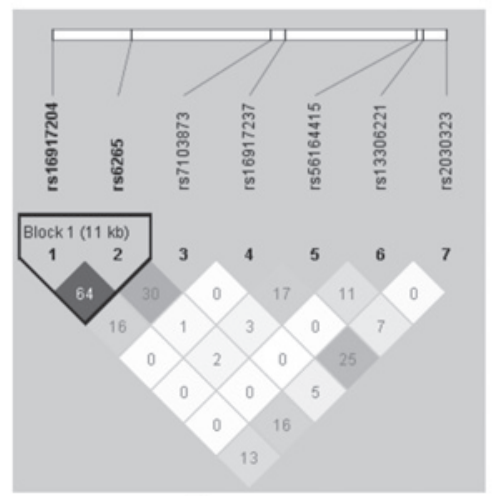

Cases $\left(\mathbf{r}^{2}\right)$

Figure 2. LD plot of the seven SNPs in the BDNF gene in controls (above) and cases (below). Values in squares are the pair-wise calculation of D' (left) or $\mathrm{r}^{2}$ (right). SNP, single nucleotide polymorphism; BDNF, brain-derived neurotrophic factor; LD, linkage disequilibrium.

exact tests or $\chi^{2}$ tests. The odds ratio (OR) and $95 \%$ confidence interval (CI) were used to measure the strength of the association between allele frequencies and the BDNF gene. Bonferroni correction was used in multiple tests and the P-value was divided by the total number of loci or haplotypes. Pair-wise linkage disequilibrium statistics ( $D^{\prime}$ and $r^{2}$ ) and haplotype frequencies were computed using Haploview 4.0 to construct haplotype blocks.

\section{Results}

Demographic and clinical characteristics. The demographic and clinical characteristics were compared between the CAD-D participants and CAD-nD participants (Table I). A higher frequency of suicide attempts and increased HAMD scores were observed in CAD-D patients than in CAD-nD participants. LD analyses of the participants and CAD-nD subjects revealed that two SNPs (rs16917204 and rs6265) were located in haplotype block 1 (D' >0.9; Fig. 2).

Genotype distribution. The genotype distribution of the seven polymorphisms was consistent with HWE. The genotype distribution and allele frequencies of the seven SNPs are listed in Table II. The distribution of haplotype frequencies is listed in Table III.

The frequency of the A allele of rs6265 was significantly higher in CAD-D patients than in the CAD-nD subjects $\left(\chi^{2}=9.634, \mathrm{P}=0.002, \mathrm{OR}=1.486,95 \% \mathrm{CI}=1.156-1.910\right.$; $\mathrm{P}<0.007$, after Bonferroni correction). Another potential association was observed for $r$ 13306221 $(P=0.019$, after 


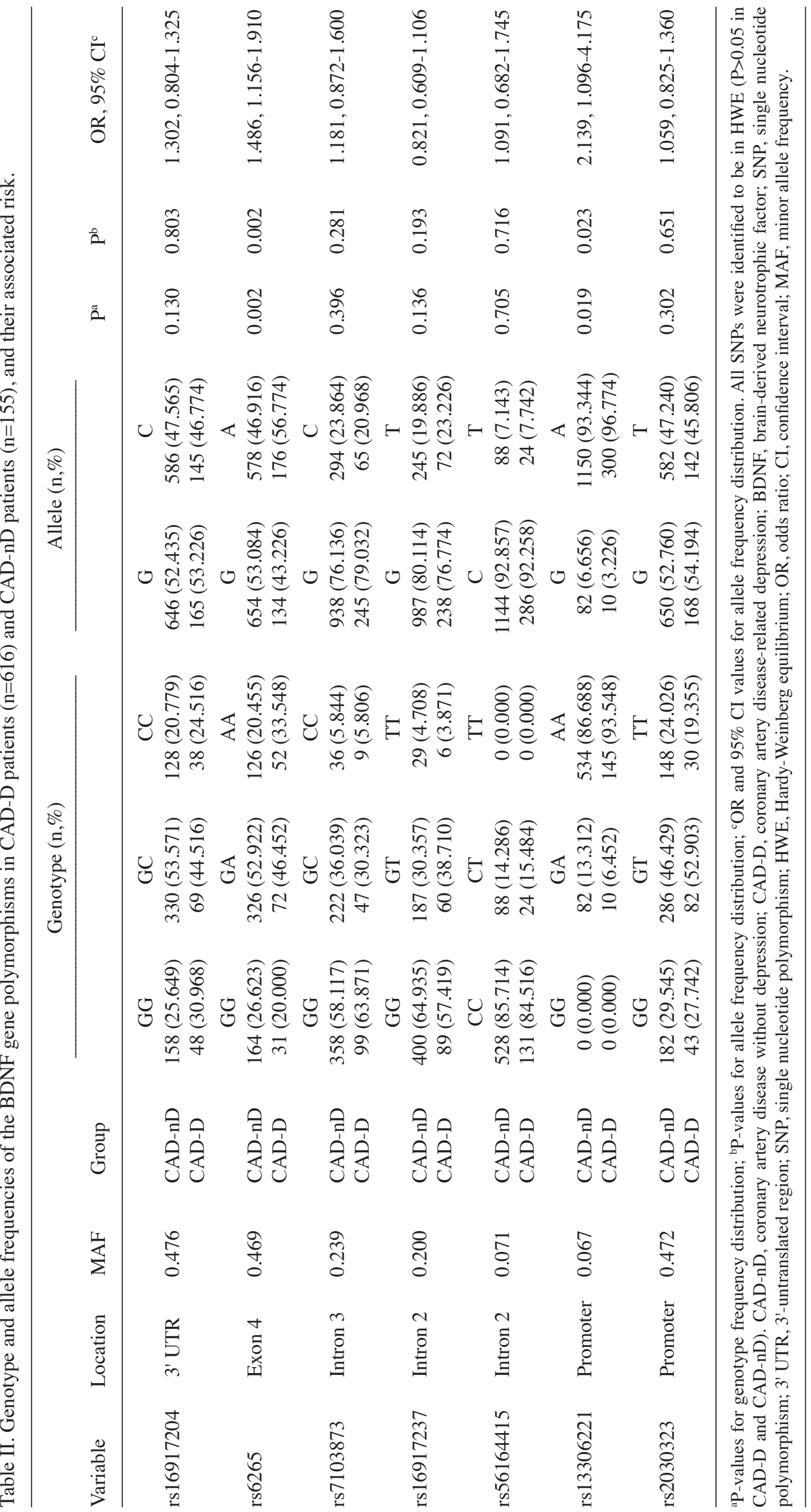


Table III. BDNF haplotype in block 1 frequencies and the results of their associations with risk of CAD-D.

\begin{tabular}{lcccccc}
\hline & & \multicolumn{4}{c}{ Statistics } \\
\cline { 5 - 7 } Haplotype $^{\mathrm{a}}$ & CAD-D (n,\%) & CAD-nD (n,\%) & $\chi^{2}$ & P & OR & $95 \%$ CI \\
\hline C-A & $72(46.45)$ & $282(45.78)$ & 0.023 & 0.881 & 1.027 & $0.722-1.463$ \\
G-G & $67(43.23)$ & $316(51.30)$ & 3.228 & 0.072 & 1.383 & $0.970-1.973$ \\
\hline
\end{tabular}

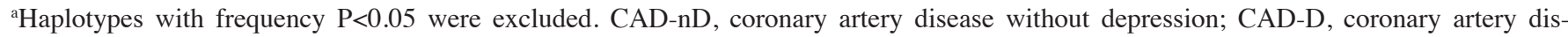
ease-related depression; BDNF, brain-derived neurotrophic factor; OR, odds ratio; CI, confidence interval.

Table IV. Genotype and allele frequency of the BDNF gene rs6265 polymorphism responded to sertraline $(\mathrm{Rs}=105$, Non-Rs=45).

\begin{tabular}{lcrr}
\hline Position & Genotype & Rs (n,\%) & Non-Rs (n,\%) \\
\hline rs6265 genotype & GG & $17(16.19)$ & $14(31.11)$ \\
& GA & $48(45.71)$ & $24(53.33)$ \\
rs6265 allele & AA & $40(38.10)$ & $7(15.56)$ \\
& G & $82(39.05)$ & $52(57.78)$ \\
& A & $128(60.95)$ & $38(42.22)$
\end{tabular}

rs6265 genotype, $\chi^{2}=8.882, P=0.012$, Rs group compared with Non-Rs group, $\mathrm{P}<0.05$ was considered to indicate a statistically significant result. rs6265 allele, $\chi^{2}=8.942, \mathrm{P}=0.003$, Rs group compared with Non-Rs group, OR=2.136, 95\% CI 1.293-3.528. BDNF, brain-derived neurotrophic factor; OR, odds ratio; CI, confidence interval.

Bonferroni correction). The CAD-D subjects had a significantly higher frequency of the $\mathrm{G}$ allele of rs13306221 but did not pass the threshold value $\left(\chi^{2}=5.194, \mathrm{P}=0.023, \mathrm{OR}=2.139\right.$, 95\% CI=1.096-4.175). Strong linkage disequilibrium was observed in block 1 (rs16917204, rs6265; D'>0.9). However, significant linkage disequilibrium was not observed between the seven SNPs in the sample population.

Correlation between rs 6265 and antidepressant treatment. We further examined the association between rs6265 and response to SSRIs. The rate of remission following the sertraline treatment was significantly higher in the subjects carrying the rs6265 A allele in CAD-D patients $\left(\chi^{2}=8.942, \mathrm{P}=0.003\right.$, $\mathrm{OR}=2.136,95 \% \mathrm{CI}=1.293-3.528$; Table IV).

\section{Discussion}

Of the 771 CAD participants in this study, 155 (20.10\%) had prominent depressive symptoms, a prevalence that is consistent with previous studies $(1,2)$. In the present study, we revealed that the A allele of rs6265 was overexpressed in CAD-D compared with CAD-nD. Following sertraline antidepressant treatment a higher rate of remission was observed in subjects carrying the rs6265 A allele. To the best of our knowledge, this is the first investigation describing evidence for a significant association between rs6265 and treatment response to sertraline in CAD-D.
Several previous studies have indicated that possession of the BDNF rs6265 A allele is a significant risk factor for MD, geriatric depression and Alzheimer's disease-related depression, and that it may modify the association between stroke and depression (16,18-21). Similarly, a recent study demonstrated that this polymorphism has been associated with CAD-D in an Italian population and that the A allele has been established as a risk factor for CAD-D (17). The present study confirmed the association of this putative functional polymorphism with CAD-D in a Chinese Han population.

The rs6265 (G196A) SNP of the BDNF gene results in the substitution of Val by Met in the 5'-pro-region of the BDNF protein at position $66(22,23)$. This substitution may evidently alter the intracellular trafficking and packaging of proBDNF, and activity dependent secretion of BDNF (24). Previous studies have suggested that carriers of the A allele may reduce hippocampal volume and increase the occurrence of MD (25). Similarly, identifying an association between the A allele in the BDNF gene and CAD-D may contribute to further elucidating the role of the BDNF gene in CAD-D.

SSRIs are commonly recommended as the first line treatment for CAD-D (26). It has been demonstrated that sertraline improved endothelial function and reduced inflammatory markers in patients with CAD and symptoms of depression (27). The rs6265 polymorphism in the BDNF gene has also been identified to be associated with the response to antidepressant medications (20). Matrisciano et al also reported that 5-week or 6-month sertraline treatment may increase the serum level of BDNF (28). Consistent with several previous studies $(29,30)$ that reported decreased release of BDNF of the rs6265 A allele, in the present study we observed that CAD-D patients with the A allele exhibited improved responses to sertraline than those with the $\mathrm{G}$ allele. This accumulative evidence suggests that the rs6265 functional polymorphism in the BDNF gene is important in the pathogenesis of at least partial CAD-D associated with decreased BDNF levels, since the antidepressant treatment evidently induced BDNF expression.

Analysis using a computational approach has suggested that the sequence of this region is potentially a part of the eukaryotic polymerase II promoter binding site and rs13306221 may disrupt the pattern of recognition, implying that rs13306221 negatively affects transcription of the BDNF gene (10). In the present study, there was a weak correlation between the SNP rs13306221 and CAD-D. The results demonstrated that CAD-D patients had a significantly higher frequency of the A allele of rs13306221 than CAD-nD subjects, although the 
frequency did not pass the threshold value. Su et al confirmed a statistically significant association between rs13306221 polymorphisms and alcohol dependence-related depression (31). Several other studies revealed that the rs13306221 polymorphism was associated with substance dependence and MD in Chinese Han population $(15,32)$. Therefore, we may reliably conclude that rs13306221 polymorphism may be involved in the pathogenesis of CAD-D by regulating BDNF transcription in our Chinese sample population. The functional importance of this polymorphism, however, requires further investigation.

In summary, genetic variations in the BDNF gene contribute to CAD-D by conferring susceptibility or resistance to antidepressant treatment. The present study further elucidates the pathogenesis of CAD-D and thus will facilitate the development of improved diagnostic and treatment strategies in CAD-D.

\section{Acknowledgements}

This study was partially supported by the National Science Foundation of China (no. NSFC31100900).

\section{References}

1. Meijer A, Conradi HJ, Bos EH, Thombs BD, van Melle JP and de Jonge P: Prognostic association of depression following myocardial infarction with mortality and cardiovascular events: a meta-analysis of 25 years of research. Gen Hosp Psychiatry 33: 203-216, 2011.

2. Thombs BD, de Jonge P, Coyne JC, Whooley MA, Frasure-Smith N, Mitchell AJ, Zuidersma M, Eze-Nliam C, Lima BB, Smith CG, Soderlund $\mathrm{K}$ and Ziegelstein RC: Depression screening and patient outcomes in cardiovascular care: a systematic review. JAMA 300: 2161-2171, 2008.

3. Lewin GR and Barde YA: Physiology of the neurotrophins. Annu Rev Neurosci 19: 289-317, 1996.

4. Donovan MJ, Lin MI, Wiegn P, Ringstedt T, Kraemer R, Hahn R, Wang S, Ibanez CF, Rafii S and Hempstead BL: Brain derived neurotrophic factor is an endothelial cell survival factor required for intramyocardial vessel stabilization. Development 127: 4531-4540, 2000.

5. Kermani P, Rafii D, Jin DK, Whitlock P, Schaffer W, Chiang A, Vincent L, Friedrich M, Shido K, Hackett NR, Crystal RG, Rafii S and Hempstead BL: Neurotrophins promote revascularization by local recruitment of TrkB+ endothelial cells and systemic mobilization of hematopoietic progenitors. J Clin Invest 115: 653-663, 2005.

6. Ejiri J, Inoue N, Kobayashi S, Shiraki R, Otsui K, Honjo T, Takahashi M, Ohashi Y, Ichikawa S, Terashima M, Mori T, Awano K, Shinke T, Shite J, Hirata K, Yokozaki H, KawashimaS and Yokoyama M: Possible role of brain-derived neurotrophic factor in the pathogenesis of coronary artery disease. Circulation 112: 2114-2120, 2005.

7. Chen B, Dowlatshahi D, MacQueen GM, Wang JF and Young LT: Increased hippocampal BDNF immunoreactivity in subjects treated with antidepressant medication. Biol Psychiatry 50: 260-265, 2001.

8. Angelucci F, Brenè S and Mathé AA: BDNF in schizophrenia, depression and corresponding animal models. Mol Psychiatry 10: 345-352, 2005.

9. Dwivedi Y, Rizavi HS, Conley RR, Roberts RC, Tamminga CA and Pandey GN: Altered gene expression of brain-derived neurotrophic factor and receptor tyrosine kinase B in postmortem brain of suicide subjects. Arch Gen Psychiatry 60: 804-815, 2003.

10. Zhang H, Ozbay F, Lappalainen J, Kranzler HR, van Dyck CH, Charney DS, Price LH, Southwick S, Yang BZ, Rasmussen A and Gelernter J: Brain derived neurotrophic factor (BDNF) gene variants and Alzheimer's disease, affective disorders, posttraumatic stress disorder, schizophrenia, and substance dependence. Am J Med Genet B Neuropsychiatr Genet 141B: 387-393, 2006.
11. Tang J, Xiao L, Shu C, Wang G, Liu Z, Wang X, Wang H and Bai X: Association of the brain-derived neurotrophic factor gene and bipolar disorder with early age of onset in mainland China. Neurosci Lett 433: 98-102, 2008.

12. Hariri AR, Goldberg TE, Mattay VS, Kolachana BS, Callicott JH, Egan MF and Weinberger DR: Brain-derived neurotrophic factor val66met polymorphism affects human memory-related hippocampal activity and predicts memory performance. J Neurosci 23: 6690-6694, 2003.

13. Borroni B, Grassi M, Archetti S, Costanzi C, Bianchi M, Caimi L, Caltagirone C, Di Luca M and Padovani A: BDNF genetic variations increase the risk of Alzheimer's disease-related depression. J Alzheimers Dis 18: 867-875, 2009.

14. Pei Y, Smith AK, Wang Y, Pan Y, Yang J, Chen Q, Pan W, Bao F, Zhao L, Tie C, Wang Y, Wang J, Zhen W, Zhou J and Ma X: The brain-derived neurotrophic-factor (BDNF) val66met polymorphism is associated with geriatric depression: a meta-analysis. Am J Med Genet B Neuropsychiatr Genet 159B: 560-566, 2012.

15. Sun RF, Zhu YS, Kuang WJ, Liu Y and Li SB: The G-712A polymorphism of brain-derived neurotrophic factor is associated with major depression but not schizophrenia. Neurosci Lett 489: 34-37, 2011.

16. Zhang L, Fang Y, Zeng Z, Lian Y, Wei J, Zhu H, Jia Y, Zhao X and Xu Y: BDNF gene polymorphisms are associated with Alzheimer's disease-related depression and antidepressant response. J Alzheimers Dis 26: 523-530, 2011.

17. Bozzini S, Gambelli P, Boiocchi C, Schirinzi S, Falcone R, Buzzi P, Storti C, Falcone C: Coronary artery disease and depression: possible role of brain-derived neurotrophic factor and serotonin transporter gene polymorphisms. Int J Mol Med 24: 813-818, 2009

18. Hwang JP, Tsai SJ, Hong CJ, Yang CH, Lirng JF and Yang YM: The Val66Met polymorphism of the brain-derived neurotrophic-factor gene is associated with geriatric depression. Neurobiol Aging 27: 1834-1837, 2006.

19. Schumacher J, Jamra RA, Becker T, Ohlraun S, Klopp N, Binder EB, Schulze TG, Deschner M, Schmäl C, Höfels S, Zobel A, Illig T, Propping P, Holsboer F, Rietschel M, Nöthen MM and Cichon S: Evidence for a relationship between genetic variants at the brain-derived neurotrophic factor (BDNF) locus and major depression. Biol Psychiatry 58: 307-314, 2005.

20. Choi MJ, Kang RH, Lim SW, Oh KS and Lee MS: Brain-derived neurotrophic factor gene polymorphism (Val66Met) and citalopram response in major depressive disorder. Brain Res 1118: 176-182, 2006.

21. Kim JM, Stewart R, Kim SW, Yang SJ, Shin IS, Kim YH and Yoon JS: BDNF genotype potentially modifying the association between incident stroke and depression. Neurobiol Aging 29: 789-792, 2008.

22. Pröschel M, Saunders A, Roses AD and Müller CR: Dinucleotide repeat polymorphism at the human gene for the brain-derived neurotrophic factor (BDNF). Hum Mol Genet 1: 353, 1992.

23. Lu B, Pang PT and Woo NH: The yin and yang of neurotrophin action. Nat Rev Neurosci 6: 603-614, 2005.

24. Egan MF, Kojima M, Callicott JH, Goldberg TE, Kolachana BS, Bertolino A, Zaitsev E, Gold B, Goldman D, Dean M, Lu B and Weinberger DR: The BDNF val66met polymorphism affects activity-dependent secretion of BDNF and human memory and hippocampal function. Cell 112: 257-269, 2003.

25. Frodl T, Schüle C, Schmitt G, Born C, Baghai T,Zill P, Bottlender R, Rupprecht R, Bondy B, Reiser M, Möller HJ and Meisenzahl EM: Association of the brain-derived neurotrophic factor Val66Met polymorphism with reduced hippocampal volumes in major depression. Arch Gen Psychiatry 64: 410-416, 2007.

26. Scherrer JF, Garfield LD, Lustman PJ, Hauptman PJ, Chrusciel T, Zeringue A, Carney RM, Freedland KE, Bucholz KK, Owen R, Newcomer JW and True WR: Antidepressant drug compliance: reduced risk of MI and mortality in depressed patients: Am J Med 124: 318-324, 2011.

27. Paraskevaidis I, Palios J, Parissis J, Filippatos G and Anastasiou-Nana M: Treating depression in coronary artery disease and chronic heart failure: what's new in using selective serotonin re-uptake inhibitors? Cardiovasc Hematol Agents Med Chem 10: 109-115, 2012.

28. Matrisciano F, Bonaccorso S, Ricciardi A, Scaccianoce S Panaccione I, Wang L, Ruberto A, Tatarelli R, Nicoletti F, Girardi P and Shelton RC: Changes in BDNF serum levels in patients with major depression disorder (MDD) after 6 months treatment with sertraline, escitalopram, or venlafaxine. J Psychiatr Res 43: 247-254, 2009. 
29. Chen ZY, Patel PD, Sant G, Meng CX, Teng KK, Hempstead BL and Lee FS: Variant brain-derived neurotrophic factor (BDNF) (Met66) alters the intracellular trafficking and activity-dependent secretion of wild-type BDNF in neurosecretory cells and cortical neurons. J Neurosci 24: 4401-4411, 2004.

30. Chen ZY, Jing D, Bath KG, Ieraci A, Khan T, Siao CJ, Herrera DG, Toth M, Yang C, McEwen BS, Hempstead BL and Lee FS: Genetic variant BDNF (Val66Met) polymorphism alters anxiety-related behavior. Science 314: 140-143, 2006.
31. Su N, Zhang L, Fei F, Hu H, Wang K, Hui H, Jiang XF, Li X, Zhen HN, Li J, Cao BP, Dang W, Qu Y and Zhou F: The brain-derived neurotrophic factor is associated with alcohol dependence-related depression and antidepressant response. Brain Res 1415: 119-126, 2011.

32. Jia W, Shi JG, Wu B, Ao L, Zhang R and Zhu YS: Polymorphisms of brain-derived neurotrophic factor associated with heroin dependence. Neurosci Lett 495: 221-224, 2011. 